\title{
Benchmarking GW against exact diagonalization for semiempirical models
}

\author{
Kaasbjerg, Kristen; Thygesen, Kristian Sommer
}

Published in:

Physical Review B Condensed Matter

Link to article, DOI:

10.1103/PhysRevB.81.085102

Publication date:

2010

Document Version

Publisher's PDF, also known as Version of record

Link back to DTU Orbit

Citation (APA):

Kaasbjerg, K., \& Thygesen, K. S. (2010). Benchmarking GW against exact diagonalization for semiempirical models. Physical Review B Condensed Matter, 81(8), 085102. https://doi.org/10.1103/PhysRevB.81.085102

\section{General rights}

Copyright and moral rights for the publications made accessible in the public portal are retained by the authors and/or other copyright owners and it is a condition of accessing publications that users recognise and abide by the legal requirements associated with these rights.

- Users may download and print one copy of any publication from the public portal for the purpose of private study or research.

- You may not further distribute the material or use it for any profit-making activity or commercial gain

- You may freely distribute the URL identifying the publication in the public portal

If you believe that this document breaches copyright please contact us providing details, and we will remove access to the work immediately and investigate your claim. 


\title{
gु \\ Benchmarking GW against exact diagonalization for semiempirical models
}

\author{
K. Kaasbjerg ${ }^{1}$ and K. S. Thygesen ${ }^{2}$ \\ ${ }^{1}$ Nano-Science Center, Niels Bohr Institute, University of Copenhagen, Universitetsparken 5, DK-2100 Copenhagen, Denmark \\ ${ }^{2}$ Center for Atomic-scale Materials Design (CAMD), Department of Physics, Technical University of Denmark, \\ DK-2800 Kongens Lyngby, Denmark
}

(Received 9 October 2009; revised manuscript received 2 December 2009; published 3 February 2010)

\begin{abstract}
We calculate ground-state total energies and single-particle excitation energies of seven pi-conjugated molecules described with the semiempirical Pariser-Parr-Pople model using self-consistent many-body perturbation theory at the GW level and exact diagonalization. For the total energies GW captures around $65 \%$ of the ground-state correlation energy. The lowest lying excitations are overscreened by GW leading to an underestimation of electron affinities and ionization potentials by $0.15 \mathrm{eV}$ on average corresponding to $\sim 3 \%$. One-shot $\mathrm{G}_{0} \mathrm{~W}_{0}$ calculations starting from Hartree-Fock reduce the screening and improve the low-lying excitation energies. The effect of the GW self-energy on the molecular excitation energies is shown to be similar to the inclusion of final-state relaxations in Hartree-Fock theory. We discuss the breakdown of the GW approximation in systems with short-range interactions (Hubbard models) where correlation effects dominate over screening/ relaxation effects. Finally we illustrate the important role of the derivative discontinuity of the true exchangecorrelation functional by computing the exact Kohn-Sham levels of benzene.
\end{abstract}

DOI: 10.1103/PhysRevB.81.085102

PACS number(s): 31.15.bu, 33.15.Ry, 31.15.V-

\section{INTRODUCTION}

For more than two decades the many-body GW approximation of Hedin ${ }^{1}$ has been the state of the art for calculating band structures of metals, semiconductors, and insulators. ${ }^{2-5}$ With the entry of nanoscience the use of the GW method has been extended to low-dimensional systems such as molecules, carbon nanotubes, graphene, and molecule-surface interfaces. ${ }^{6-13}$ In these systems the interplay between quantum confinement (in one or more dimensions) and electronic correlation effects leads to distinct phenomena such as the renormalization of molecular electronic levels at surfaces by dynamical polarization in the substrate. ${ }^{11-15}$ Very recently, the nonequilibrium version of the GW approximation has been applied to quantum transport and dynamics in molecular junctions ${ }^{16-23}$ where dynamic correlations seems to be particularly important.

As the range of systems to which the GW approximation is being applied continues to expand, critical investigations of the performance of GW for other systems than the crystalline solids become important. Here we report on benchmark GW calculations for $\pi$-conjugated molecules based on the semiempirical Pariser-Parr-Pople (PPP) model. ${ }^{24-26}$ By comparing with exact results we obtain a direct and unbiased estimate of the quality of the GW approximation in molecular systems.

Previous benchmark model studies of the GW approximation have all focused on Hubbard models with short-range (local) interactions described by the Hubbard $U .^{22,27-29}$ The conclusion from these studies is that GW works well in the small $U$ regime but fails for medium-strong interactions strengths. The use of GW in systems with local interactions is in fact unfortunate because the importance of electronic screening, which is the main effect described by GW, is weak in comparison to correlation effects. In contrast to Hubbard models, the PPP description includes long-range interactions and its parameters have been fitted to yield realis- tic excitation energies of conjugated molecules. It therefore provides a better and more natural starting point for a study addressing the accuracy of GW for real molecules and nanostructures. We mention that in a related work we have performed first-principles GW calculations for a series of 34 molecules arriving at very similar conclusions regarding the performance of $\mathrm{GW}$ as those reported here. ${ }^{30}$

$A b$ initio GW calculations typically involve a number of "technical" approximations such as the plasmon pole approximation, the neglect of off-diagonal matrix elements in the GW self-energy, or analytic continuations. Moreover they are usually performed non-self-consistently and are subject to basis set errors. In the present work the GW calculations are carried out fully self-consistently without any further approximations apart from the $\mathrm{GW}$ approximation itself.

In this work we calculate total energies and excitation spectra of the seven conjugated molecules listed in Table I. The excitation spectrum of a system can be obtained from the spectral function

TABLE I. Chemical formula, number of $p_{z}$ orbitals $(L)$ included in the PPP model, exact ground-state entropies $(S)$ of the reduced single-particle density matrix, and exact HOMO-LUMO gaps for the listed molecules.

\begin{tabular}{lcccc}
\hline \hline & Formula & $L$ & $S / S_{\max }$ & $\begin{array}{c}E_{\text {gap }} \\
(\mathrm{eV})\end{array}$ \\
\hline Thiophene & $\mathrm{C}_{4} \mathrm{H}_{4} \mathrm{~S}$ & 5 & 0.07 & 11.19 \\
Pyridine & $\mathrm{C}_{5} \mathrm{H}_{5} \mathrm{~N}$ & 6 & 0.11 & 10.61 \\
Benzene & $\mathrm{C}_{6} \mathrm{H}_{6}$ & 6 & 0.10 & 11.39 \\
Benzene (Hubbard) & & & 0.50 & \\
Biphenyl & $\mathrm{C}_{12} \mathrm{H}_{10}$ & 12 & 0.10 & 9.24 \\
Naphthalene & $\mathrm{C}_{10} \mathrm{H}_{8}$ & 10 & 0.11 & 8.65 \\
Anthracene & $\mathrm{C}_{14} \mathrm{H}_{10}$ & 14 & 0.12 & 7.06 \\
OPV2 & $\mathrm{C}_{14} \mathrm{H}_{12}$ & 14 & 0.10 & 8.30 \\
\hline \hline
\end{tabular}




$$
\begin{aligned}
A_{i}(\varepsilon)= & 2 \pi \sum_{n}\left|\left\langle\Psi_{n}(N+1)\left|c_{i}^{\dagger}\right| \Psi_{0}(N)\right\rangle\right|^{2} \delta\left(\varepsilon-\varepsilon_{n}\right) \\
& +2 \pi \sum_{n}\left|\left\langle\Psi_{n}(N-1)\left|c_{i}\right| \Psi_{0}(N)\right\rangle\right|^{2} \delta\left(\varepsilon-\varepsilon_{n}\right),
\end{aligned}
$$

which has peaks at the excitation energies $\varepsilon_{n}=E_{n}(N+1)$ $-E_{0}(N)$ and $\varepsilon_{n}=E_{0}(N)-E_{n}(N-1)$ corresponding to electronic addition and removal energies, respectively. Often in the GW literature, excitation energies are referred to as quasiparticle (QP) energies. In the expressions for the excitation energies $E_{n}(N)$ denotes the energy of the $n$th excited $N$-electron state, $\left|\Psi_{n}(N)\right\rangle$, with $N$ referring to the neutral state of the system. For molecules the first addition and the first removal energy, i.e., $n=0$, corresponds to the electron affinity and the ionization potential. In Hartree-Fock (HF) theory Koopman's theorem ${ }^{31}$ states that the eigenvalues of the Hartree-Fock Hamiltonian equal the addition/removal energies calculated without orbital relaxations in the charged states, i.e., $\varepsilon_{n}^{\mathrm{HF}}=\left\langle c_{n}^{\dagger} \Psi_{0}^{\mathrm{HF}}(N)|H| c_{n}^{\dagger} \Psi_{0}^{\mathrm{HF}}(N)\right\rangle-E_{0}^{\mathrm{HF}}(N)$ for a virtual orbital $n$. In particular, the highest occupied molecular orbital (HOMO) and the lowest unoccupied molecular orbital (LUMO) represent well-defined approximations to the ionization potential and electron affinities, respectively. ${ }^{32}$ This approximation neglects two important effects. One is the relaxation of the single-particle HF orbitals when an electron is removed from or added to the molecule. The other is the correlation energy which by definition is omitted in $\mathrm{HF}$ theory. It is instructive to write the exact QP energies as the sum of the three contributions

$$
\varepsilon_{n}=\varepsilon_{n}^{\mathrm{HF}}+\Delta_{\text {relax }}+\Delta_{\text {corr }} .
$$

The relaxation contribution is the correction that follows by calculating the QP energy from self-consistently determined $\mathrm{HF}$ energies of the neutral and the charged states $N \pm 1$. The last term $\Delta_{\text {corr }}$ is the remaining contribution from the correlation energy. For the addition of an electron, i.e., an unoccupied orbital, the relaxation, and correlation contributions are given by

$$
\Delta_{\text {relax }}=E_{n}^{\mathrm{HF}}(N+1)-E_{0}^{\mathrm{HF}}(N)-\varepsilon_{n}^{\mathrm{HF}}
$$

and

$$
\Delta_{\text {corr }}=\left[E_{n}(N+1)-E_{n}^{\mathrm{HF}}(N+1)\right]-\left[E_{0}(N)-E_{0}^{\mathrm{HF}}(N)\right] .
$$

In extended systems the potential due to a single delocalized electron/hole decreases with the size of the system. Hence, in such systems there will be no or little relaxation of the states due to the addition/removal of an electron and the majority of the correction to the QP energy will come from the correlation part $\Delta_{\text {corr }}$. In molecules, nanostructures, molecules at surfaces, and disordered systems with finite localization lengths, this is not the case. Here, the introduction of an additional electron or hole will lead to a relaxation of the single-particle orbitals corresponding to a screening of the additional charge. As a consequence, the relaxation correction $\Delta_{\text {relax }}$ to the QP energy cannot be neglected in such systems. In fact, we find that $\Delta_{\text {relax }}$ is larger than $\Delta_{\text {corr }}$ for all the molecules studied here and that the GW excitation energies correspond roughly to including only $\Delta_{\text {relax }}$ in Eq. (2).
The paper is outlined as follows. In Sec. II the PPP model Hamiltonian for conjugated molecules is introduced. In Secs. III A and III B we provide an overview of the theory and numerical implementation of the GW and exact calculations, and in Sec. III C we discuss the use of the von Neumann entropy as a measure of correlation. The results for total energies and spectral properties of the PPP model are presented in Secs. IV A and IV B, and a comparison is made to short ranged Hubbard models in Sec. IV C. In Sec. IV D we calculate the exact Kohn-Sham (KS) levels for the benzene molecule and compare to the exact QP levels. The conclusions are given in Sec. V.

\section{PARISER-PARR-POPLE HAMILTONIAN}

The Pariser-Parr-Pople model is an effective $\pi$-electron description of conjugated molecules that includes electronelectron interactions explicitly. The PPP Hamiltonian is given by

$$
\begin{aligned}
H= & \sum_{i} \varepsilon_{i} \hat{n}_{i}-\sum_{\langle i j\rangle \sigma} t_{i j} c_{i \sigma}^{\dagger} c_{j \sigma} \\
& +\frac{1}{2} \sum_{i \neq j} V_{i j}\left(\hat{n}_{i}-Z_{i}\right)\left(\hat{n}_{j}-Z_{j}\right)+\sum_{i} U_{i} \hat{n}_{i \uparrow} \hat{n}_{i \downarrow},
\end{aligned}
$$

where $c_{i}^{\dagger}\left(c_{i}\right)$ creates (annihilates) an electron in the $p_{z}$ orbital on atom $i$ of the molecule, $\hat{n}_{i}=\hat{n}_{i \uparrow}+\hat{n}_{i \downarrow}$ is the number operator, $\hat{n}_{i \sigma}=c_{i \sigma}^{\dagger} c_{i \sigma}, Z_{i}$ is the valence (i.e., the number of $\pi$ electrons) of atom $i$, and $\langle i j\rangle$ denotes nearest-neighbor hopping. The Ohno parametrization ${ }^{33}$ is used for the long-range interactions

$$
V_{i j}=\frac{14.397}{\sqrt{\left[28.794 /\left(U_{i}+U_{j}\right)\right]^{2}+R_{i j}^{2}}},
$$

where $R_{i j}$ is the interatomic distance (in $\AA$ ) and $U_{i}$ is the on-site Coulomb interaction (in $\mathrm{eV}$ ). For large distances the Ohno parametrization recovers the $1 / r$ behavior of the Coulomb interaction while it for small distances represents a screened interaction that interpolates to on-site Coulomb interaction $U_{i}$ for $R_{i j}=0$. The on-site energy $\varepsilon_{i}$, the hopping element $t_{i j}$, and the on-site Coulomb interaction $U_{i}$ are treated as fitting parameters. In the present work values for these parameters have been taken from the literature. ${ }^{34-38}$ Since existing parameters have been optimized to optical excitation spectra, an exact agreement with experimental values for the molecular gaps is not to be expected.

\section{METHODS}

\section{A. GW approximation}

Hedin's equations ${ }^{1}$ provides a formally exact framework for the determination of the single-particle Green's function in a self-consistent manner. In the GW approximation, which follows by neglecting the so-called vertex corrections, the electronic self-energy $\Sigma$ is given by the product of the Green's function $G$ and the screened interaction $W$, and can be written symbolically as 


$$
\Sigma=i G W,
$$

where the Green's function obeys the usual Dyson equation $G=G_{0}+G_{0} \Sigma G$. The screened interaction $W$ is given by the bare Coulomb interaction $V$ and the polarization in the random-phase approximation $P=-i G G$ through the Dysontype equation

$$
W=V+V P W .
$$

In fully self-consistent $\mathrm{GW}$ the set of coupled equations for $\Sigma, G, P$, and $W$ are solved iteratively until the Green's function has converged. Due to the computational requirement of a fully self-consistent $\mathrm{GW}$ scheme, ab initio $\mathrm{GW}$ calculations are usually carried out non-self-consistently. This approach, which is referred to as $G_{0} W_{0}$, starts from an approximate $G_{0}$, typically the noninteracting Kohn-Sham Green's function, from which a single self-energy iteration is carried out to obtain the final Green function.

\section{Numerical details}

The GW calculations have been performed following the method described in detail in Ref. 39. Here we give a brief overview of the method for completeness.

The retarded and advanced single-particle Green's functions are given by

$$
G^{r / a}(\varepsilon)=\left[\varepsilon \pm i \eta-H_{0}-V_{\mathrm{H}}-\Sigma_{\mathrm{GW}}(\varepsilon)\right]^{-1},
$$

where $\eta$ is a small positive infinitesimal, $H_{0}$ contains the first two terms in Eq. (5), and $V_{\mathrm{H}}$ is the Hartree potential. We represent the Green's functions and all other energydependent quantities on a uniform grid, $-E_{m},-E_{m}$ $+d \varepsilon, \ldots, E_{m}$. The fast Fourier transform is used to switch between the energy and time representations. Since $\eta$ determines the minimum width of features in the Green's function's energy dependence, the energy grid spacing should obey $d \varepsilon \ll \eta$. All results presented here have been converged with respect to $\eta, d \varepsilon, E_{m}$. Typical converged values are (in eV) $\eta=0.02, d \varepsilon=0.005, E_{m}=50$.

The lesser/greater Green's functions are given by

$$
\begin{gathered}
G^{<}(\varepsilon)=-f(\varepsilon-\mu)\left[G^{r}-G^{a}\right], \\
G^{>}(\varepsilon)=[1-f(\varepsilon-\mu)]\left[G^{r}-G^{a}\right],
\end{gathered}
$$

where $f(\varepsilon-\mu)$ is the Fermi-Dirac function. The chemical potential $\mu$ is adjusted to yield the desired number of electrons in the system. The formulation in terms of a fixed chemical potential rather than a fixed particle number is reminiscent of the fact that the method has been developed for quantum transport. The one-body density matrix is given by

$$
\rho_{i j}=-i \int G_{i j}^{<}(\varepsilon) d \varepsilon .
$$

From $\rho$ the Hartree and exchange potentials follow

$$
\begin{gathered}
V_{\mathrm{H}, i j}=\delta_{i j} 2 \sum_{k} V_{i k} \rho_{k k}, \\
V_{x, i j}=-V_{i j} \rho_{i j},
\end{gathered}
$$

where we have defined $V_{i i}=U_{i}$, see Eq. (5).
The retarded/advanced and lesser/greater components of the quantities needed to construct the GW self-energy read ${ }^{39}$

$$
\begin{aligned}
\Sigma_{\mathrm{GW}, i j}^{<,>}(t) & =i G_{i j}^{<,>}(t) W_{i j}^{<,>}(t), \\
W_{i j}^{<,>}(\varepsilon) & =\sum_{k l} W_{i k}^{r}(\varepsilon) P_{k l}^{<,>}(\varepsilon) W_{l j}^{a}(\varepsilon), \\
W_{i j}^{r, a}(\varepsilon) & =\sum_{k} P_{i k}^{r, a}\left[1-V P^{r, a}(\varepsilon)\right]_{k j}^{-1}, \\
P_{i j}^{<,>}(t) & =-i G_{i j}^{<,>}(t) G_{j i}^{>,<}(-t) .
\end{aligned}
$$

The GW equations have been expressed in the time or energy domain according to where they are simplest. This also reflects the practical implementation.

The retarded components of $\Sigma_{\mathrm{GW}}$ and $P$ are obtained using the fundamental relation

$$
F^{r}(t)=-i \theta(t)\left[F^{>}(t)-F^{<}(t)\right] .
$$

Since the GW self-energy depends on the Green's function and vice versa, the equations must be iterated until selfconsistency. To speed up convergence we use the Pulay mixing scheme ${ }^{40}$ as described in Ref. 39.

\section{Total energy}

The total energy can be split into kinetic (and external), Hartree, and exchange-correlation (xc) energy $E=E_{0}+E_{\mathrm{H}}$ $+E_{\mathrm{xc}}$. In terms of the Green's function we have

$$
E_{0}+E_{\mathrm{H}}=\operatorname{Tr}\left[H_{0} \rho\right]+\frac{1}{2} \operatorname{Tr}\left[V_{\mathrm{H}} \rho\right] .
$$

For the exchange-correlation energy we have

$$
E_{\mathrm{xc}}=\frac{1}{2 i} \int \operatorname{Tr}\left[\Sigma^{r}(\varepsilon) G^{<}(\varepsilon)+\Sigma^{<}(\varepsilon) G^{a}(\varepsilon)\right] d \varepsilon,
$$

where $\Sigma$ is the exchange-correlation self-energy. In this work $\Sigma$ is either the bare exchange, $\Sigma_{x}$, yielding the HF approximation, or the GW self-energy, $\Sigma_{\mathrm{GW}}$. The expression (21) follows by expressing $\langle\hat{V}\rangle$ in terms of the two-particle Green's function, $G_{2}$, and then using the defining equation for the self-energy in terms of $G_{2}{ }^{41}$

\section{B. Exact diagonalization}

The most direct way to the spectral properties of a system is via the Lehmann representation of the Green's function in Eq. (1). However, since this requires the full set of eigenstates and eigenvalues of the Hamiltonian, it is of limited practical use and other routes must be taken. The following section gives a brief overview of the Lanczos method for iterative diagonalization of large matrices.

\section{Calculating the ground state-Lanczos algorithm}

In exact diagonalization the given many-body Hamiltonian is diagonalized directly in the Fock space which is spanned by many-particle states (Slater determinants). Since 
the dimensionality of the Fock space grows exponentially with the number of basis orbitals, symmetries of the Hamiltonian can help to reduce the dimensionality considerably. For the Pariser-Parr-Pople Hamiltonian in Eq. (5) the number of up and down electrons, $N_{\uparrow}$ and $N_{\downarrow}$, are good quantum numbers since their corresponding operators commute with the Hamiltonian. This implies that the exact diagonalization can be carried out in each of the $\left(N_{\uparrow}, N_{\downarrow}\right)$ subblocks of the Fock space independently. The dimensionality of each $\left(N_{\uparrow}, N_{\downarrow}\right)$ subblock is given by the number of ways $N_{\uparrow}$ spin-up electrons and $N_{\downarrow}$ spin-down electrons can be distributed over $L$ basis orbitals,

$$
d\left(N_{\uparrow}, N_{\downarrow}\right)=\frac{L !}{N_{\uparrow} !\left(L-N_{\uparrow}\right) !} \times \frac{L !}{N_{\downarrow} !\left(L-N_{\downarrow}\right) !} .
$$

Very often the ground state is located in the half-filled subblock, i.e., $N_{\uparrow}=N_{\downarrow}=L / 2$ where $L$ is the number of basis orbitals. For $L=16$ the dimensionality of this subblock is $d$ $=165636900$, implying that storing a vector in double floating point precision requires $\sim 1 \mathrm{~Gb}$ of memory. With such memory requirements a full diagonalization of the Hamiltonian is of course out of reach. If only the ground state is needed, iterative methods can be employed. The basic idea of iterative methods is to project the Hamiltonian onto the Krylov subspace $\mathcal{K}$ generated by repeated applications of $H$ on an arbitrary initial state $\left|\phi_{0}\right\rangle$, i.e.,

$$
\mathcal{K}=\operatorname{span}\left\{\left|\phi_{0}\right\rangle, H\left|\phi_{0}\right\rangle, H^{2}\left|\phi_{0}\right\rangle, \ldots, H^{N}\left|\phi_{0}\right\rangle\right\} .
$$

In the Krylov subspace the extreme eigenvalues of the Hamiltonian converge fast with respect to the size $N$ of the subspace, thus reducing the full diagonalization to a manageable diagonalization of a $N \times N$ matrix with $N \ll d$.

In the Lanczos algorithm ${ }^{42}$ the Hamiltonian is projected onto a specially constructed orthogonalized Krylov basis in which the Hamiltonian has a tridiagonal representation. The basis vectors are generated recursively as

$$
\left|\phi_{n+1}\right\rangle=H\left|\phi_{n}\right\rangle-a_{n}\left|\phi_{n}\right\rangle-b_{n}^{2}\left|\phi_{n-1}\right\rangle,
$$

where the coefficient are given by

$$
a_{n}=\frac{\left\langle\phi_{n}|H| \phi_{n}\right\rangle}{\left\langle\phi_{n} \mid \phi_{n}\right\rangle} \quad \text { and } \quad b_{n}^{2}=\frac{\left\langle\phi_{n} \mid \phi_{n}\right\rangle}{\left\langle\phi_{n-1} \mid \phi_{n-1}\right\rangle}
$$

with initial conditions $b_{0}=0$ and $\left|\phi_{-1}\right\rangle=0$. At any point during the Lanczos iterations only three Lanczos vectors needs to be kept in memory, which makes the algorithm memory efficient. In the basis of the normalized vectors (the basis vectors above are not normalized) the Hamiltonian has the following tridiagonal representation:

$$
H=\left(\begin{array}{ccccc}
a_{0} & b_{1} & 0 & \cdots & 0 \\
b_{1} & a_{1} & b_{2} & & \vdots \\
0 & b_{2} & a_{2} & \ddots & 0 \\
\vdots & & \ddots & \ddots & b_{N} \\
0 & \cdots & 0 & b_{N} & a_{N}
\end{array}\right),
$$

which can be readily diagonalized with methods for tridiagonal matrices. In practice the Lanczos iterations are continued until the desired eigenvalues have converged to a given tol- erance. For the ground-state energy $E_{0}$, typical values for $N$ range from a few to $\sim 200$ depending on the system size.

The ground state resulting from a diagonalization of the tridiagonal Hamiltonian in Eq. (26) is provided in the Lanczos basis, i.e., $\left|\Psi_{0}\right\rangle=\Sigma_{n} c_{n}\left|\phi_{n}\right\rangle$. In order to be able to calculate the Green's function, its representation in the original manybody basis is required. Since the Lanczos vectors are not stored, the Lanczos iterations must be repeated (starting from the same initial vector) to obtain the expansion coefficients $\alpha_{i}=\Sigma_{n} c_{n}\left\langle\Phi_{i} \mid \phi_{n}\right\rangle$ in the original many-body basis $\left\{\left|\Phi_{i}\right\rangle\right\}_{i=1}^{d}$.

The most time consuming part of the Lanczos algorithm is the matrix-vector multiplication $H\left|\phi_{n}\right\rangle$. An efficient implementation of this part is hence crucial. For this purpose it is convenient to use the bit representation of an unsigned integer to code the basis states. Denoting the integers with bit representations corresponding to the spin-up and spin-down occupations of a given basis state with $I_{\uparrow}$ and $I_{\downarrow}$, respectively, the integer representation of the basis state is $I=I_{\uparrow}+2^{L} I_{\downarrow}$. With the binary representation of the basis states, the multiplication of the Hamiltonian can be done efficiently using bitwise operations.

\section{Calculating the Green's function}

Having obtained the ground state, the Green's function can now be calculated. From the Lehmann representation it follows that it can be written as

$$
G_{i j}^{r}(\varepsilon)=G_{i j}^{e}(\varepsilon)+G_{i j}^{h}(\varepsilon)
$$

with the electron and hole Green's functions defined by

$$
G_{i j}^{e}(\varepsilon)=\left\langle\Psi_{0}^{N}\left|c_{i} \frac{1}{\varepsilon-H+E_{0}^{N}+i \eta} c_{j}^{\dagger}\right| \Psi_{0}^{N}\right\rangle
$$

and

$$
G_{i j}^{h}(\varepsilon)=\left\langle\Psi_{0}^{N}\left|c_{j}^{\dagger} \frac{1}{\varepsilon+H-E_{0}^{N}+i \eta} c_{i}\right| \Psi_{0}^{N}\right\rangle,
$$

respectively. In the following we focus on the electron Green's function which is the matrix representation of the resolvent operator $(z-H)^{-1}$ in the basis spanned by the $|i\rangle$ $=c_{i}^{\dagger}\left|\Psi_{0}^{N}\right\rangle$ vectors. To obtain the $i$ th diagonal element,

$$
G_{i i}^{e}(\varepsilon)=\left\langle i\left|(z-H)^{-1}\right| i\right\rangle,
$$

where $z=\varepsilon+E_{0}^{N}+i \eta$, again the Lanczos algorithm is used to put $H$ on a tridiagonal form but this time the Lanczos iterations are started from the normalized initial state $\left|\phi_{0}\right\rangle$ $=|i\rangle / b_{0}$, where $b_{0}^{2}=\langle i \mid i\rangle$. Hence, in the generated Krylov subspace the diagonal element in Eq. (30) corresponds to the matrix element $b_{0}^{2}\left[\left(\varepsilon-H+E_{0}^{N}+i \eta\right)^{-1}\right]_{11}$ of a tridiagonal matrix, which can be obtained as the continued fraction ${ }^{43}$

$$
G_{i i}^{e}(\varepsilon)=\frac{b_{0}^{2}}{\varepsilon-a_{0}-\frac{b_{1}^{2}}{\varepsilon-a_{1}-\frac{b_{2}^{2}}{\varepsilon-a_{2}-\cdots}} .} .
$$

Again the Lanczos iterations are continued until the frequency dependent Green's function element has converged. 


\section{C. von Neumann entropy}

The following section demonstrates how a quantitative measure of the degree of correlations in a system can be obtained by considering the von Neumann entropy of the reduced single-particle density matrix $\rho$. The entropy is defined by

$$
S[\rho]=-\operatorname{Tr}[\rho \log \rho]=-\sum_{n} \rho_{n} \log \rho_{n},
$$

where in the last equality $\rho$ has been expressed in its diagonal representation, $\rho=\Sigma_{n} \rho_{n}|n\rangle\langle n|$.

In the basis of the atomic $p_{z}$ orbitals the matrix elements of the reduced density matrix are given by (with the spin index suppressed)

$$
\rho_{i j}=\left\langle\Psi_{0}\left|c_{j}^{\dagger} c_{i}\right| \Psi_{0}\right\rangle
$$

with the diagonal elements equal to the site occupations. In the diagonal representation $\rho_{n}$ thus represents the occupation of the eigenstate $|n\rangle$ of the density matrix.

We note that $0 \leq S \leq S_{\max }$, with $S_{\max }=L \log 2$, and $2 L$ is the dimension of the single-particle Hilbert space including spin. The expression for $S_{\max }$ follows because the number of electrons equal $L$ in all the systems, i.e., half-filled "band." When $\left|\Psi_{0}\right\rangle$ is a single Slater determinant (corresponding to zero correlation) we have $S=0$ and when $\left|\Psi_{0}\right\rangle$ has equal weight on a complete set of orthogonal Slater determinants (corresponding to maximal correlation) we have $\rho_{n}=1 / 2$ for all $n$ and thus $S=L \log 2$. Thus the number $0 \leq S / S_{\max } \leq 1$ represents a natural measure of the degree of correlation in $\left|\Psi_{0}\right\rangle$.

\section{RESULTS}

\section{A. Total energies}

We first address the degree of correlation in the exact ground states by considering the von Neumann entropies of the corresponding density matrices. The calculated entropies are listed in Table I. Except for the Hubbard description of benzene (see Sec. IV C) which clearly presents strong correlations, the entropies of the ground states are $\sim 10 \%$ of their maximum value $S_{\max }$ corresponding to weakly correlated systems. The finite values of the entropies reveal that none of the ground states are single Slater determinants implying that the Hartree-Fock ground-state energies will be larger than the exact ones.

We here follow the usual convention and define the correlation energy as the part of the total energy not included in Hartree-Fock, i.e.,

$$
E_{\text {corr }}=E_{\text {exact }}-E_{\mathrm{HF}} \text {. }
$$

Figure 1 shows the exact correlation energies of the neutral molecules together with those obtained by evaluating the total energy from Eqs. (20) and (21) with the self-consistently determined Green's function and GW self-energy.

For the series of molecules considered here the correlation energy constitute less than $0.5 \%$ of the total energies. Furthermore, as expected it increases (in absolute size) with increasing number of atoms in the molecule. Clearly, the GW

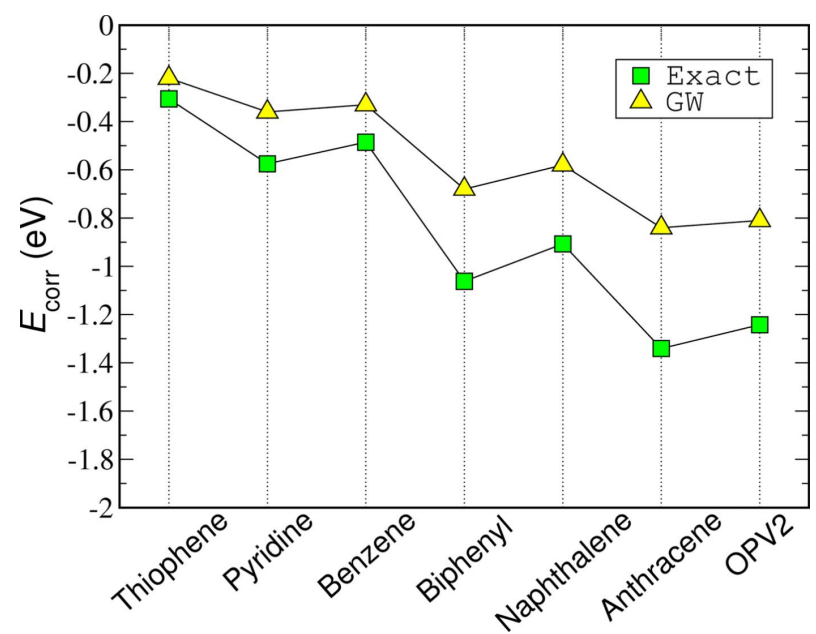

FIG. 1. (Color online) Exact and GW correlation energies of the neutral ground state of the seven molecules.

approximation performs reasonably well for all the molecules capturing on average $66 \%$ of the correlation energy.

\section{B. Spectral properties}

For isolated systems such as molecules, true quasiparticles resembling single-particle excitations are characterized by having a weight close to unity (for nondegenerate levels) in the spectral function, i.e.,

$$
Z_{n}=\sum_{i}\left|\left\langle\Psi_{n}^{N+1}\left|c_{i}^{\dagger}\right| \Psi_{0}^{N}\right\rangle\right|^{2} \sim 1
$$

This is equivalent to saying that there exists an orbital $|\nu\rangle$ so that the excited state $\left|\Psi_{n}^{N+1}\right\rangle$ can be written as the singleparticle excitation $c_{\nu}^{\dagger}\left|\Psi_{0}^{N}\right\rangle$. In Fig. 2 we show the singleparticle density of states (DOS),

$$
D(\varepsilon)=\sum_{i} A_{i}(\varepsilon)
$$

for the OPV2 molecule on a logarithmic scale. The height of the peaks reflects the value of $Z_{n}$ (modulo degeneracies). The

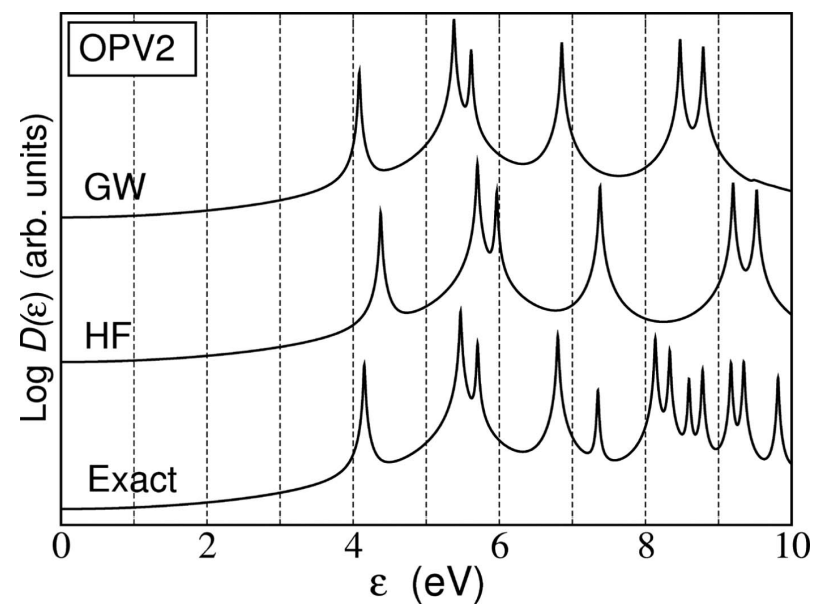

FIG. 2. Single-particle DOS of the OPV2 molecule. Note the logarithmic axis. 


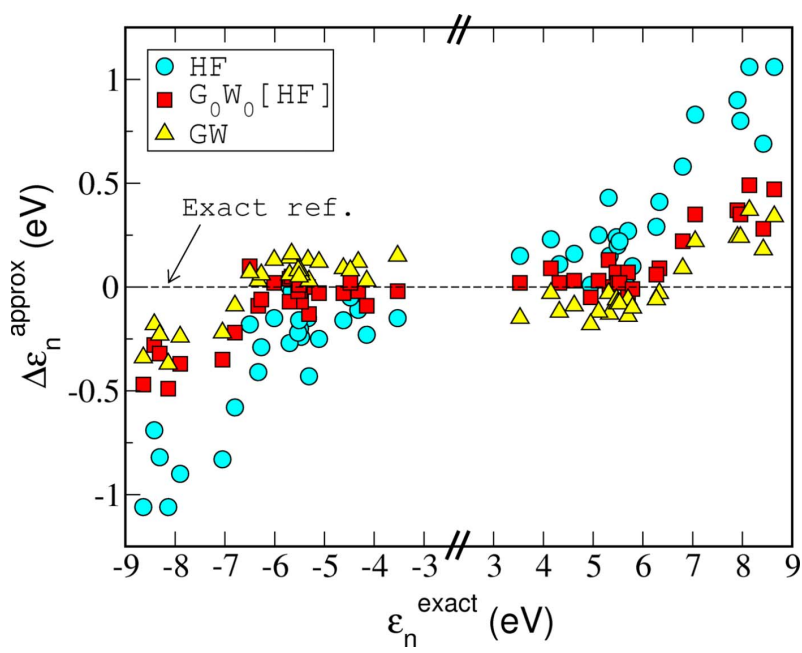

FIG. 3. (Color online) Energy of the three highest occupied and three lowest unoccupied molecular orbitals relative to the exact values. While Hartree-Fock underestimates the occupied and overestimates the unoccupied levels, self-consistent GW shows the opposite trends but deviates on average less from the exact result.

HF and, in particular, the GW approximation reproduce the lowest lying excitations quite well while higher excitations are poorly described. All the peaks in the HF spectrum have $Z_{n}=1$ while $\mathrm{GW}$ does shift some spectral weight from the main peaks to tiny satellite structures (at higher energies than shown on the plot). However, the GW satellites do not correspond to features in the exact spectrum. This shows that excitations with $Z_{n} \ll 1$, i.e., excitations which do not have single-particle character, are not well described by $\mathrm{GW}$ whose main effect is to improve the position of the HF single-particle peaks.

In the following we consider the lowest lying singleparticle excitations of the molecules as obtained with Hartree-Fock, $\mathrm{G}_{0} \mathrm{~W}_{0}$ and self-consistent $\mathrm{GW}$. In the $\mathrm{G}_{0} \mathrm{~W}_{0}$ calculations the starting Green's function $G_{0}$ is taken to be the self-consistently determined Hartree-Fock Green's function. Figure 3 gives an overview of the calculated excitation energies relative to the exact ones. Energies corresponding to electron removal and electron addition are located on the negative and positive half of the $x$ axis, respectively. From this plot clear trends in the calculated excitation energies emerge.

Within HF the occupied (unoccupied) levels are systematically overestimated (underestimated) and the deviation from the exact values worsens for the higher lying excitations.

Both the $\mathrm{G}_{0} \mathrm{~W}_{0}$ and the $\mathrm{GW}$ give consistently better energies than HF-in particular, for the higher lying excitations where the absolute errors are reduced to less than $\sim 0.4 \mathrm{eV}$ as compared to $\sim 1 \mathrm{eV}$ for HF. For the low-lying excitations GW slightly overestimates (underestimates) the occupied (unoccupied) levels corresponding to an overcorrection of the HF energies.

In order to address the relative contributions from $\Delta_{\text {relax }}$ and $\Delta_{\text {corr }}$ to the excitation energies in Eq. (2), we plot in Fig. 4 the difference between the exact gaps and the gaps obtained from the (i) Hartree-Fock eigenvalues, (ii) Hartree-

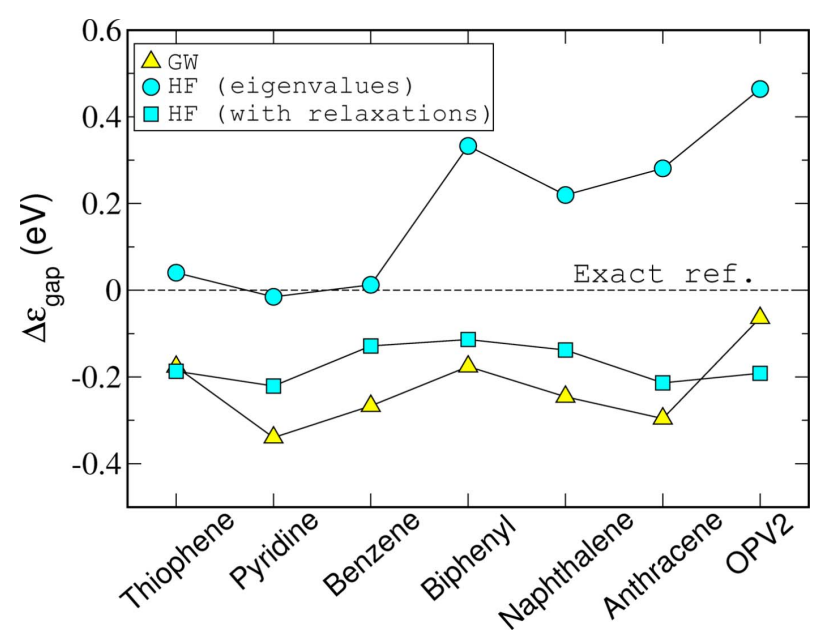

FIG. 4. (Color online) The HOMO-LUMO gap relative to the exact values. In addition to the HF and GW single-particle energies, the relaxed Hartree-Fock total-energy differences, $E_{0}^{\mathrm{HF}}(N+1)$ $+E_{0}^{\mathrm{HF}}(N-1)-2 E_{0}^{\mathrm{HF}}(N)$ are also shown. The excellent results of $\mathrm{HF}$ for the three smallest molecules is a result of error cancellation between relaxation and correlation contributions.

Fock total-energy differences with self-consistent relaxations in the $N \pm 1$ Slater determinants taken into account, and (iii) the distance between the highest occupied and lowest unoccupied peaks in the GW spectral function. By using the expression for the quasiparticle energies in Eq. (2), the exact gap $E_{\text {gap }}=\varepsilon_{\mathrm{LUMO}}-\varepsilon_{\mathrm{HOMO}}$ can be expressed as

$$
E_{\text {gap }}=\varepsilon_{\mathrm{LUMO}}^{\mathrm{HF}}-\varepsilon_{\mathrm{HOMO}}^{\mathrm{HF}}+\Delta_{\text {relax }}^{\text {gap }}+\Delta_{\text {corr }}^{\text {gap }},
$$

where $\Delta_{\text {relax }}^{\text {gap }}$ and $\Delta_{\text {corr }}^{\text {gap }}$ are the gap equivalents of the corresponding quantities in Eq. (2) and $\varepsilon_{\mathrm{HOMO} / \mathrm{HF} O}^{\mathrm{HF}}$ are the Hartree-Fock HOMO/LUMO eigenvalues. By definition $\Delta_{\text {relax }}^{\text {gap }}$ is the difference between the gaps obtained from the HF eigenvalues and relaxed HF total-energy differences. In Fig. 4 this is given by the vertical distance between the (blue) squares and circles. The correlation contribution $\Delta_{\text {corr }}^{\text {gap }}$ can be read off as the difference between the exact gap (dashed horizontal line) and the relaxed HF total-energy gap (blue squares). Inclusion of relaxation effects clearly reduces the HF gaps considerably implying that $\Delta_{\text {relax }}^{\text {gap }}<0$. This reduction is due to the screening from the orbital relaxation which reduces the Coulomb interaction with the added hole or electron and hence also the gap.

We note that the HF eigenvalues give excellent gaps for the small single-ring molecules thiophene, pyridine, and benzene. The good agreement with the exact levels for these systems is not a result of HF giving a correct description of the many-body states and their energies - this was already clear from the analysis above which showed that the eigenstates are not single Slater determinants. Hence the excitation energies in Eq. (2) have contributions from both $\Delta_{\text {relax }}$ and $\Delta_{\text {corr }}$. The good agreement must therefore be ascribed to cancellations between the relaxation and correlation contribution to the exact energies.

In contrast to the HF (eigenvalue) gaps for which the agreement with the exact gap worsens as a function of the size of the molecules, the GW gaps follow more consistently 


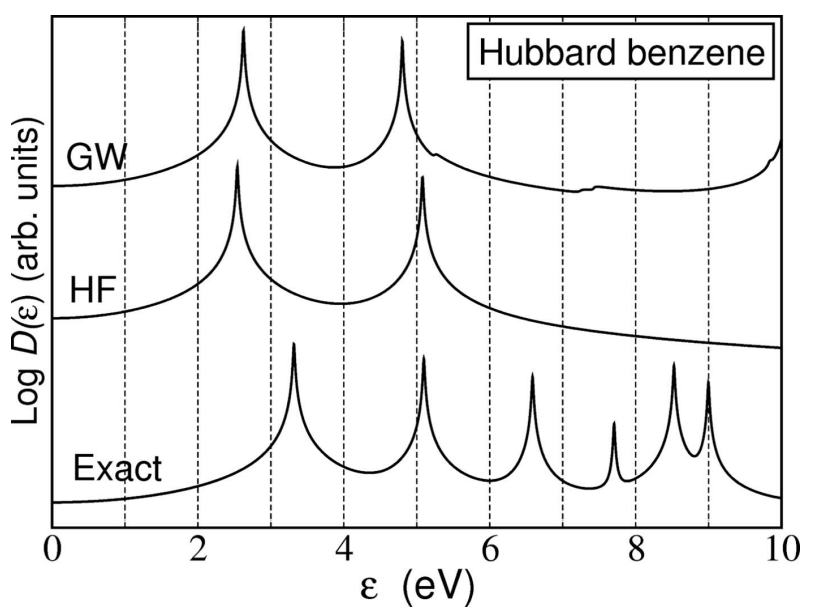

FIG. 5. Single-particle DOS for the Hubbard description of the benzene molecule (only on-site interactions from the PPP model are kept). Note the logarithmic axis.

the same trend and underestimates the exact gaps with 0.05$0.35 \mathrm{eV}$ for all the molecules. The close resemblance between GW and the relaxed HF result indicates that the effect of GW is mainly to account for the screening effects included in HF via orbital relaxations, $\Delta_{\text {relax }}$.

\section{Long-range versus short-range interactions}

To demonstrate the shortcomings of the GW approximation for strongly correlated systems, we consider a Hubbard model description of the benzene molecule. It should be noted that this Hubbard description of benzene is not intended as a realistic description of the benzene molecule, rather it serves to illustrate the limitations of the GW approximation. The Hamiltonian is identical to the PPP Hamiltonian in Eq. (5), except that the long-range Coulomb interactions in the third term have been omitted. The values for the hopping elements and the on-site Coulomb interaction are $t=2.539$ and $U=10.06$, respectively. With a $U / t$ ratio of $\sim 4$ this obviously represent a strongly correlated system. The latter is reflected in the ground-state entropy in Table I which is $50 \%$ of its maximum value.

From the calculated total energies we find that the correlation energy (not included in Fig. 1) constitutes 10\% of the ground-state energy which is a considerably higher fraction as for the PPP descriptions of the molecules. The GW total energy captures $88 \%$ of the correlation energy compared to $66 \%$ on the average for the PPP descriptions. However, from an absolute point of view, the GW approximation misses the exact ground-state energy by $0.48 \mathrm{eV}$. This should be compared to $0.16 \mathrm{eV}$ which is the difference between the exact and the GW ground-state energy for the PPP description of benzene.

The poor performance of both Hartree-Fock and GW for the spectral properties of the Hubbard benzene is illustrated in Fig. 5 which shows the spectral function as calculated with the two methods together with the exact one. Both Hartree-Fock and GW severely underestimate the position of the LUMO level and completely misses the details of the spectrum at higher energies. This clearly demonstrates that
$\mathrm{GW}$ is of limited relevance when considering systems where correlation effects $\left(\Delta_{\text {corr }}\right)$ dominates over screening, or relaxation, effects $\left(\Delta_{\text {relax }}\right)$.

\section{Exact Kohn-Sham orbital energies}

Within density-functional theory (DFT) the eigenvalues of the single-particle Kohn-Sham Hamiltonian, $H_{\mathrm{KS}}$, are often interpreted as physical energies. In principle the validity of this approximation depends on the size of the derivative discontinuity of the true and unknown xc functional. ${ }^{44,45}$ In practice the use of semilocal xc functionals represents an additional approximation. It is of general interest to investigate to what extent the discrepancy between KS energies and true QP energies result from the use of approximate functionals and to what extent this is a property of the exact functional. Below we compare the exact Kohn-Sham spectrum to the exact QP spectrum of the PPP benzene molecule using the lattice version of DFT.

The lattice version of DFT follows by extending the fundamental concepts of standard DFT, such as the HohenbergKohn theorem and the Kohn-Sham equations, to model Hamiltonians as, e.g., the PPP Hamiltonian. ${ }^{46}$ In this reformulation of DFT the site occupations $n_{i}$ replaces the continuous electron density $n(\mathbf{r})$ as the fundamental variable that determines the ground-state properties. The lattice version of the single-particle Kohn-Sham Hamiltonian is given by the sum of the hopping terms (the kinetic energy) and a site dependent Kohn-Sham potential $V_{i}^{\mathrm{KS}}$ which is constructed to yield the correct site occupations of the ground state,

$$
H_{\mathrm{KS}}=-\sum_{\langle i j\rangle \sigma} t_{i j} c_{i \sigma}^{\dagger} c_{j \sigma}+\sum_{i} V_{i}^{\mathrm{KS}} \hat{n}_{i} .
$$

For the present purpose the explicit form of the site potential $V_{i}^{\mathrm{KS}}$ is not important. The fact that the lattice version of the Kohn-Sham potential is an on-site potential and is equivalent to the restriction of the Kohn-Sham potential $V_{\mathrm{xc}}(\mathbf{r})$ in the real-space formulation of DFT to a local potential.

Due to the high symmetry of the benzene molecule all sites in the PPP Hamiltonian are equivalent implying that $V_{i}^{\mathrm{KS}}$ has the same value for all sites. Except for a constant shift, the eigenvalues of the Kohn-Sham Hamiltonian are therefore given by those of the hopping part of the Hamiltonian. The HOMO-LUMO gap calculated from the KohnSham eigenvalues is $E_{\text {gap }}^{\mathrm{KS}}=5.08 \mathrm{eV}$ which is a severe underestimation of the true gap of $11.39 \mathrm{eV}$. In line with previous studies $^{44,45}$ we thus conclude that the main reason for the discrepancy between KS eigenvalues obtained with approximate xc functionals and the exact orbital energies is due to the derivative discontinuity of the exact xc functional.

\section{CONCLUSION}

We have presented calculations for the total energy and charged single-particle excitations in seven conjugated molecules described by the semiempirical PPP model within fully self-consistent GW and exact diagonalization. The results show that the GW approximation gives a consistently good description of both total energies and electronic excita- 
tions with a slight tendency to overestimate (underestimate) the position of the latter for occupied (unoccupied) levels. We have found that the effect of the GW self-energy is similar to the inclusion of orbital relaxations in the $N \pm 1$ final states in Hartree-Fock theory. On the other hand the contribution to the excitation energies coming from correlations in the ground state and excited state is less well described by GW. This explains why GW tend to reduce electron addition/ removal energies relative to the $\mathrm{HF}$ eigenvalues. It was shown that GW does not perform well for systems with short-range interactions (Hubbard models) where correlation effects are dominating over screening/relaxation effects. Finally it was shown that the exact Kohn-Sham eigenvalues significantly underestimate the true HOMO-LUMO gap of a benzene molecule showing the importance of the derivative discontinuity of the exact exchange-correlation functional.

\section{ACKNOWLEDGMENTS}

Financial support from the Danish Council for Production and Technology (FTP) under Grant No. 26-04-0181 "Atomic-scale modeling of emerging electronic devices" is acknowledged. K.S.T. acknowledges support from the Danish Center for Scientific Computing. The Center for Atomicscale Materials Design (CAMD) is sponsored by the Lundbeck Foundation.
${ }^{1}$ L. Hedin, Phys. Rev. 139, A796 (1965).

${ }^{2}$ M. S. Hybertsen and S. G. Louie, Phys. Rev. B 34, 5390 (1986).

${ }^{3}$ W. G. Aulbur, L. Jonsson, and J. W. Wilkins, in Solid State Physics, edited by H. Ehrenreich and F. Saepen (Academic, New York, 2000), Vol. 54, p. 1.

${ }^{4}$ F. Aryasetiawan and O. Gunnarsson, Rep. Prog. Phys. 61, 237 (1998).

${ }^{5}$ G. Onida, L. Reining, and A. Rubio, Rev. Mod. Phys. 74, 601 (2002).

${ }^{6}$ J. C. Grossman, M. Rohlfing, L. Mitas, S. G. Louie, and M. L. Cohen, Phys. Rev. Lett. 86, 472 (2001).

${ }^{7}$ A. Stan, N. E. Dahlen, and R. van Leeuwen, Europhys. Lett. 76, 298 (2006).

${ }^{8}$ C. D. Spataru, S. Ismail-Beigi, L. X. Benedict, and S. G. Louie, Phys. Rev. Lett. 92, 077402 (2004).

${ }^{9}$ P. E. Trevisanutto, C. Giorgetti, L. Reining, M. Ladisa, and V. Olevano, Phys. Rev. Lett. 101, 226405 (2008).

${ }^{10}$ M. L. Tiago and J. R. Chelikowsky, Phys. Rev. B 73, 205334 (2006).

${ }^{11}$ J. B. Neaton, M. S. Hybertsen, and S. G. Louie, Phys. Rev. Lett. 97, 216405 (2006).

${ }^{12}$ C. Freysoldt, P. Rinke, and M. Scheffler, Phys. Rev. Lett. 103, 056803 (2009).

${ }^{13}$ J. M. Garcia-Lastra, C. Rostgaard, A. Rubio, and K. S. Thygesen, Phys. Rev. B 80, 245427 (2009).

${ }^{14}$ K. Kaasbjerg and K. Flensberg, Nano Lett. 8, 3809 (2008).

${ }^{15}$ K. S. Thygesen and A. Rubio, Phys. Rev. Lett. 102, 046802 (2009).

${ }^{16}$ C. D. Spataru, L. X. Benedict, and S. G. Louie, Phys. Rev. B 69, 205204 (2004).

${ }^{17}$ K. S. Thygesen and A. Rubio, J. Chem. Phys. 126, 091101 (2007).

${ }^{18}$ P. Darancet, A. Ferretti, D. Mayou, and V. Olevano, Phys. Rev. B 75, 075102 (2007).

${ }^{19}$ K. S. Thygesen, Phys. Rev. Lett. 100, 166804 (2008).

${ }^{20}$ P. Myöhänen, A. Stan, G. Stefanucci, and R. van Leeuwen, EPL 84, 67001 (2008).

${ }^{21}$ C. D. Spataru, M. S. Hybertsen, S. G. Louie, and A. J. Millis, Phys. Rev. B 79, 155110 (2009).

${ }^{22}$ M. Puig von Friesen, C. Verdozzi, and C.-O. Almbladh, Phys. Rev. Lett. 103, 176404 (2009).

${ }^{23}$ N. E. Dahlen and R. van Leeuwen, Phys. Rev. Lett. 98, 153004
(2007).

${ }^{24}$ J. A. Pople, Trans. Faraday Soc. 49, 1375 (1953).

${ }^{25}$ R. Pariser and R. G. Parr, J. Chem. Phys. 21, 466 (1953).

${ }^{26}$ R. Pariser and R. G. Parr, J. Chem. Phys. 21, 767 (1953).

${ }^{27}$ A. Schindlmayr, T. J. Pollehn, and R. W. Godby, Phys. Rev. B 58, 12684 (1998).

${ }^{28}$ T. J. Pollehn, A. Schindlmayr, and R. W. Godby, J. Phys.: Condens. Matter 10, 1273 (1998).

${ }^{29}$ C. Verdozzi, R. W. Godby, and S. Holloway, Phys. Rev. Lett. 74, 2327 (1995).

${ }^{30}$ C. Rostgaard, K. W. Jacobsen, and K. S. Thygesen, Phys. Rev. B 81, 085103 (2010).

${ }^{31} \mathrm{P}$. Fulde, Electron Correlations in Molecules and Solids, Springer Series in Solid-State Sciences Vol. 100, 3rd ed. (Springer, Berlin, 1995).

${ }^{32}$ In the remainder of the paper HOMO and LUMO abbreviations will be used to refer to the ionization potential and electron affinity also outside Hartree-Fock theory.

${ }^{33}$ K. Ohno, Theor. Chim. Acta 2, 219 (1964).

${ }^{34}$ I. R. Ducasse, T. E. Miller, and Z. G. Soos, J. Chem. Phys. 76, 4094 (1982).

${ }^{35}$ W. Barford and R. J. Bursill, Chem. Phys. Lett. 268, 535 (1997).

${ }^{36}$ R. J. Bursill, C. Castleton, and W. Barford, Chem. Phys. Lett. 294, 305 (1998).

${ }^{37}$ W. Barford, R. J. Bursill, and M. Y. Lavrentiev, J. Phys.: Condens. Matter 10, 6429 (1998).

${ }^{38}$ M. Yu. Lavrentiev, W. Barford, S. J. Martin, H. Daly, and R. J. Bursill, Phys. Rev. B 59, 9987 (1999).

${ }^{39}$ K. S. Thygesen and A. Rubio, Phys. Rev. B 77, 115333 (2008).

${ }^{40}$ P. Pulay, Chem. Phys. Lett. 73, 393 (1980).

${ }^{41}$ A. L. Fetter and J. D. Walecka, Quantum Theory of ManyParticle Systems (McGraw-Hill, New York, 1971).

${ }^{42}$ The present work has used the implementation from the IETL project (Ref. 47).

${ }^{43}$ E. Dagotto, Rev. Mod. Phys. 66, 763 (1994).

${ }^{44}$ J. P. Perdew and M. Levy, Phys. Rev. Lett. 51, 1884 (1983).

${ }^{45}$ R. W. Godby, M. Schluter, and L. J. Sham, Phys. Rev. Lett. 56, 2415 (1986).

${ }^{46}$ K. Schonhammer, O. Gunnarsson, and R. M. Noack, Phys. Rev. B 52, 2504 (1995).

${ }^{47} \mathrm{P}$. Dayal, M. Troyer, and $\mathrm{R}$. Villiger, The Iterative Eigensolver Template Library (IETL), http://www.compphys.org/software/ietl/ 